\title{
Incorporating Domain Knowledge into Medical NLI using Knowledge Graphs
}

\author{
Soumya Sharma* \\ soumyasharma20 $0^{\dagger}$
}

T Y S S Santosh

santoshtyss ${ }^{\dagger}$

\author{
Bishal Santra* \\ bsantraigi ${ }^{\dagger}$
}
Niloy Ganguly
niloy

\author{
Abhik Jana \\ abhikjana ${ }^{\dagger}$
}

\author{
Pawan Goyal \\ pawang ${ }^{\ddagger}$
}

\author{
Indian Institute of Technology Kharagpur, India \\ $\{\dagger\}$ @gmail.com, $\{\ddagger\} @$ cse.iitkgp.ac.in
}

\begin{abstract}
Recently, biomedical version of embeddings obtained from language models such as BioELMo have shown state-of-the-art results for the textual inference task in the medical domain. In this paper, we explore how to incorporate structured domain knowledge, available in the form of a knowledge graph (UMLS), for the Medical NLI task. Specifically, we experiment with fusing embeddings obtained from knowledge graph with the state-of-theart approaches for NLI task, which mainly rely on contextual word embeddings. We also experiment with fusing the domain-specific sentiment information for the task. Experiments conducted on MedNLI dataset clearly show that this strategy improves the baseline BioELMo architecture for the Medical NLI $\operatorname{task}^{1}$.
\end{abstract}

\section{Introduction}

Natural language inference (NLI) is one of the basic natural language understanding tasks which deals with detecting inferential relationship such as entailment or contradiction, between a given premise and a hypothesis. In recent years, with the availability of large annotated datasets like SNLI (Bowman et al., 2015), MultiNLI (Williams et al., 2018), researchers have come up with several neural network based models which could be trained with these large annotated datasets and are able to produce state-of-the-art performances (Bowman et al., 2015, 2016; Munkhdalai and Yu, 2017; Sha et al., 2016; Chen et al., 2017;

\footnotetext{
eqqual contribution

${ }^{1}$ https://github.com/soummyaah/ KGMedNLI/
}

Tay et al., 2018). With these attempts, even though NLI in domains like fiction, travel etc. has progressed a lot, NLI in medical domain is yet to be explored extensively. With the introduction of MedNLI (Romanov and Shivade, 2018), an expert annotated dataset for NLI in the clinical domain, researchers have started pursuing the problem of clinical NLI. Modeling informal inference is one of the basic tasks towards achieving natural language understanding, and is considered very challenging. MedNLI is a dataset that assists in assessing how good a sentence or word embedding method is for downstream uses in medical domain.

Recently, with the emergence of advanced contextual word embedding methods like ELMo (Peters et al., 2018) and BERT (Devlin et al., 2018), performances of many NLP tasks have improved, setting state-of-the-art performances. Following this stream of literature, Lee et al. (2019) introduce BioBERT, which is a BERT model pretrained on English Wikipedia, BooksCorpus and fine-tuned on PubMed (7.8B tokens in total) corpus, PMC full-text articles. Jin et al. (2019) propose BioELMo which is a domain-specific version of ELMo trained on 10M PubMed abstracts, and attempt to solve medical NLI problem with these domain specific embeddings, leading to state-ofthe-art performance. These two attempts show a direction towards solving medical NLI problem where the pretrained embeddings are fine-tuned on medical corpus and are used in the state-of-the-art NLI architecture.

Chen et al. (2018) proposed the use of external knowledge to help enrich neural-network based NLI models by applying Knowledge-enriched coattention, Local inference collection with Exter- 
nal Knowledge, and Knowledge-enchanced inference composition components. Another line of solution tries to bring in the extra domain knowledge from sources like Unified Medical Language System (UMLS) (Bodenreider, 2004). Romanov and Shivade (2018) used the knowledge-directed attention based methods in (Chen et al., 2018) for Medical NLI. Another such attempt is made by Lu et al. (2019), where they incorporate domain knowledge in terms of the definitions of medical concepts from UMLS with the state-of-the-art NLI model ESIM (Chen et al., 2017) and vanilla word embeddings of Glove (Pennington et al., 2014) and fastText (Bojanowski et al., 2017). Even though, the authors achieve significant improvement by incorporating only concept definitions from UMLS, the features of this clinical knowledge graph are yet to be fully utilized. Motivated by the emerging trend of embedding knowledge graphs to encode useful information in a high dimensional vector space, we propose the idea of applying state-of-the-art knowledge graph embedding algorithm on UMLS and use these embeddings as a representative of additional domain knowledge with the state-of-the-art medical NLI models like BioELMo, to investigate the performance improvement on this task. Additionally, we also incorporate the sentiment information for medical concepts given by MetaMap (Aronson and Lang, 2010) leading to further improvement of the performance. Note that, as state-of-theart baselines, we use the models proposed by Jin et al. (2019) and Lu et al. (2019). Since, both of these studies consider ESIM as the core NLI model which makes it more convenient for us to incorporate extra domain knowledge and to have a fair performance comparison with these state-ofthe-art models. Our contributions are two-fold.

- We incorporate domain knowledge via knowledge graph embeddings applied on UMLS. We propose an intelligent path-way to combine contextual word embeddings with the domain specific embeddings learnt from the knowledge graph, and feed them to the state-of-the-art NLI models like ESIM. This helps to improve the performance of the base architecture.

- We further show the usefulness of the associated sentiments per medical concept from UMLS in boosting the performance further, which in a way shows that if we can carefully use the domain knowledge present in sources like UMLS, it can lead to promising results as far as the medical NLI task is concerned.

\section{Dataset}

In this study, we use the MedNLI dataset (Romanov and Shivade, 2018), a well-accepted dataset for natural language inference in clinical domain. The dataset is sampled from doctors' notes in the clinical dataset MIMIC-III (Alistair EW Johnson and Mark., 2016) and is arguably the largest publicly available database of patient records. The entire dataset consists of 14,049 premise-hypothesis pairs divided into 11,232 train pairs, 1,395 validation pairs and 1,422 test pairs. Each such pair consists of a gold label which could be either entailment (true), contradiction (false), or neutral (undetermined). The average (maximum) sentence lengths of premises and hypotheses are 20 (202) and $5.8(20)$, respectively.

\section{Proposed Approach}

The task is to classify the given premise $(p)$ and hypothesis $(h)$ sentence pair into one of the three classes: entailment, contradiction and neutral. Following the approach by Jin et al. (2019), we use the BioELMo embedding model where authors bring in contextual information in terms of embeddings obtained via applying ELMo trained on 10M PubMed abstracts, and use these with the state-of-the-art ESIM model (Chen et al., 2017) for the NLI task. The architecture includes two sentence encoders each of which takes in as input the word embeddings of $p$ and $h$. The inputs are run through corresponding bi-directional LSTM encoder layers. Pairwise attention matrix is computed between encoded $p$ and $h$, which forms the attention layer followed by a second bi-directional LSTM layer run separately over $p$ and $h$. Max and average pooling are performed over the outputs of LSTM layers and the output of pooling operations is run through a softmax model. We feed this architecture an additional domain knowledge from UMLS as vector representations obtained via knowledge graph embeddings, the details of which are described below.

UMLS: Unified Medical Language System (UMLS) is a compendium which includes many health and biomedical vocabularies and standards. It provides a mapping structure between these 


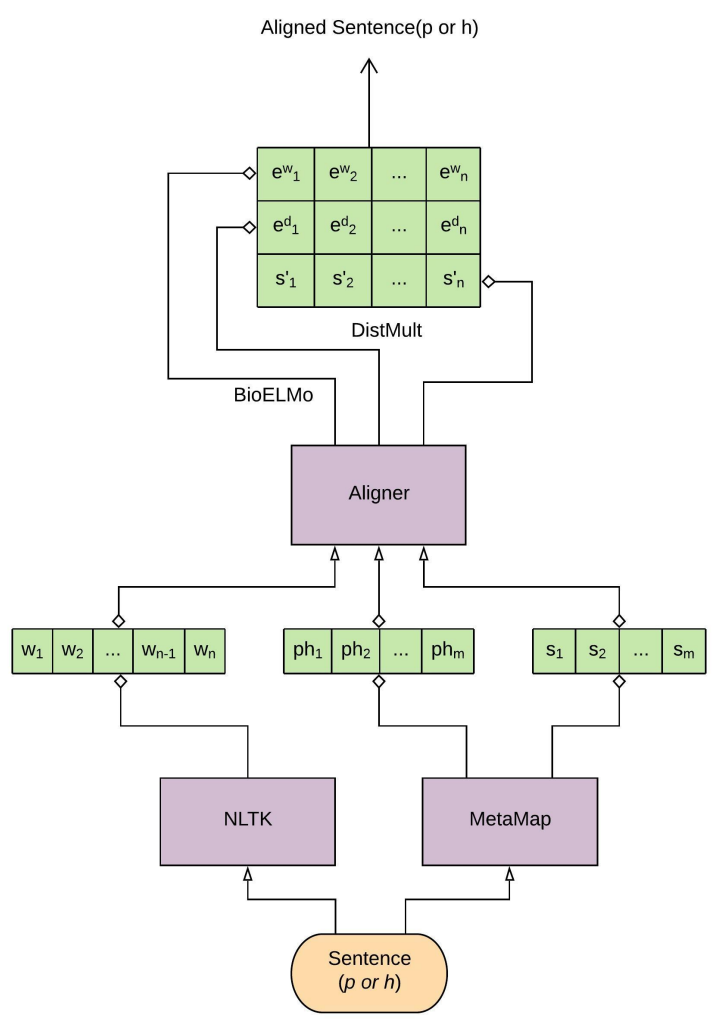

Figure 1: BioELMo w/ KG pipeline Here $w$ is the NLTK tokenized form of premise ( $\mathrm{p}$ ) or hypothesis (h), $p h$ is the MetaMap tokenized form of sentence ( $\mathrm{p}$ or h). $s$ signifies the sentiment vector. $e^{w}$ and $e^{d}$ signify the aligned word embeddings and DistMult embeddings, respectively. $s^{\prime}$ signifies the aligned sentiment vector.

vocabularies and is a comprehensive thesaurus and ontology of biomedical concepts. UMLS contains 3 knowledge sources: Metathesaurus, Semantic Network, and Specialist Lexicon and Lexical Tools. We use two of these sources: the Metathesaurus and the Semantic Network. The Metathesaurus comprises of over 1 million biomedical concepts and 5 million concept names. Each concept has numerous relationships with each other. Each concept in the Metathesaurus is assigned one or more Semantic Type linked to other Semantic Types through a semantic relationship. This information is provided in the Semantic Network of UMLS. There are 127 semantic types and 54 relationships in total. Semantic types include "disease", "symptom", "laboratory test" and semantic relationships include "is-a", "part-of", "affects".

MetaMap: MetaMap is a tool for effective entity mapping of biomedical text to the concepts and

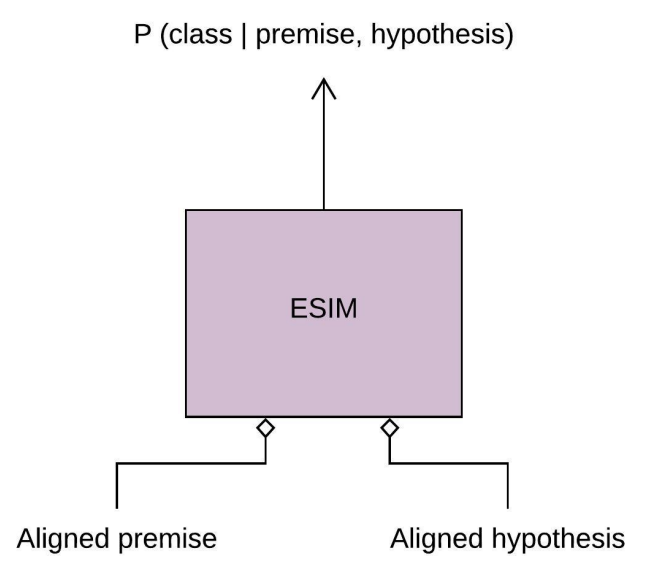

Figure 2: Aligned premise and hypothesis embeddings are obtained using the method described in Figure 1 and become inputs to the ESIM model.

semantic type in UMLS Metathesaurus. On feeding a sentence to MetaMap, it divides the sentence into phrases based on medical concepts found in the sentence and for each medical concept it provides its unique ID in Metathesaurus, its position in the sentence, the list of semantic types the concept is mapped to, the preferred medical name and unique ID for the preferred concept (such as a concept called "chest pain" would be mapped to its preferred medical term "angina"). We also get a boolean value associated with each concept denoting whether the medical concept occurs in a negative sentiment (1) or not (0). For example, in the sentence, "The patient showed no signs of pain", the medical concept 'pain' would appear with a negative sentiment. Note that, for each extracted phrase, there may be more than one related medical concepts and each concept may have more than one possible mapping. For our study, we only consider the mapping with the highest MetaMap Indexing (MMI) score, a metric provided by MetaMap. As a result, every word in a sentence has zero or one corresponding medical concept.

Constructing the appropriate knowledge graph: We use the MetaMap tool to process the complete MedNLI dataset and extract the relevant information from UMLS into a smaller knowledge graph.

First, we use MetaMap to extract medical concepts from $p$ and $h$, and map them to the standard terminology in UMLS. We choose to map each medical concept to its preferred medical term. 
E.g., "blood clots" would map to "thrombus". This helps us to map different synonymous surface forms to the same concept. This results in 7,496 unique medical concepts from UMLS matched to various words and phrases in the MedNLI dataset. Each unique concept in UMLS becomes a node in our knowledge graph. The relations in our knowledge graph come from two sources: The Metathesaurus and the Semantic Network of UMLS.

Using relations extracted from these two sources, we connect the filtered medical concepts from UMLS to build a smaller Knowledge Graph (subgraph of UMLS).

We get 117,467 triples from the Metathesaurus and 23,824,105 triples from the Semantic Network, which constitute the edgelists in the prepared knowledge graph.

Knolwedge Graph Embeddings: To obtain the embedding from this graph, we use state-ofthe-art DistMult model (Bishan Yang and Deng, 2015). The choice is inspired by Kadlec et al. (2017), which reports that an appropriately tuned DistMult model can produce similar or better performance while compared with the competing knowledge graph embedding models. DistMult model embeds entities (nodes) and relations (edges) as vectors. It uses a matrix dot product to measure compatibility between head $(h)$ and tail $(t)$ entities, connected by a relation $(r)$. Logistic loss is used for training the model.

$$
\sigma_{\text {DistMult }}(h, r, t)=r^{T}(h \cdot t)
$$

Combining Knolwedge Graph Embeddings with BioELMo: As explained in Figure 1, each sentence $(p$ or $h)$ is tokenized using the simple module of NLTK $^{2}$ as well as processed using MetaMap to get UMLS concepts. To align these, we copy the UMLS concept for a phrase to all the constituent words.

Once we have aligned the tokens obtained via NLTK and MetaMap, we apply BioELMo and DistMult to get the embedding vectors, $e_{\text {BioELMo,w }}$ and $e_{\text {DistMult }, w}$ for each word $w$. We concatenate these vectors as $e_{w}=e_{B i o E L M o, w} \oplus$ $e_{\text {DistMult,w }}$, to obtain the word representation for $w$. We call the proposed model which uses these embeddings as BioELMo w/KG.

Combining Sentiment Information: We further enhance the domain knowledge by incorporating sentiment information for a concept sepa-

\footnotetext{
${ }^{2}$ https://www.nltk.org/
}

rately. For that purpose, we use the sentiment boolean provided by MetaMap and create a 1-d vector $\left(\right.$ sent $\left._{w}\right)$ containing 0 for positive medical concepts or non-medical concept and 1 for negative concept. This 1-d vector is aligned with the $e_{\text {DistMult,w }}$ in the same fashion as explained above. We concatenate this single dimension with our concatenated resultant embeddings. Thus $e_{w}=e_{B i o E L M o, w} \oplus e_{\text {DistMult }, w} \oplus$ sent $_{w}$. We call the proposed model which uses these embeddings as BioELMo w/ KG + Sentiment.

We use the vanilla ESIM model (Chen et al., 2017) and feed the obtained concatenated embeddings for each word in the premise and hypothesis to the model, to be trained for the inference task (see Figure 2).

\begin{tabular}{ll}
\hline Model & Accuracy \\
\hline fastText & $68.7 \%$ \\
GloVe & $73.1 \%$ \\
BioELMo (Jin et al., 2019) & $78.2 \%$ \\
ESIMw/K (Lu et al., 2019) & $77.8 \%$ \\
\hline fastText w/ KG+Sentiment & $73.67 \%$ \\
GloVe w/ KG+Sentiment & $74.46 \%$ \\
BioELMo w/ KG & $78.76 \%$ \\
BioELMo w/ KG+Sentiment & $\mathbf{7 9 . 0 4 \%}$ \\
\hline
\end{tabular}

Table 1: Performance of our models (bottom four) along with the state-of-the-art baseline models (top four). Baseline results for fastText, GloVe are obtained from Romanov and Shivade (2018). Adding

knowledge graph information to the base models showed an absolute improvement of $4.97 \%$ in case of fastText and $1.36 \%$ in case of GloVe. The baseline model utilizing BioELMo as base embeddings (Jin et al., 2019) showed an accuracy of $78.2 \%$. On adding knowledge graph information, we were able to improve these results to $78.76 \%$ and on further addition of sentiment information, the accuracy rose to $79.04 \%$

\section{Experimental Results and Analysis}

As discussed earlier, we mainly consider the models presented by Jin et al. (2019) [BioELMo] and Lu et al. (2019) $[E S I M w / K]$ as our baselines. We report accuracy as the performance metric. Table 1 represents the performance comparison of our proposed models and the baselines, which shows that incorporation of knowledge graph embeddings helps to improve the model performance. All the results reported use ESIM as their base model. Further, incorporating sentiment of medical concepts gives further improvements, 
achieving an overall $1 \%$ improvement over the baseline model.

We also see from (Jin et al., 2019) that BERT and BioBERT show an accuracy of $77.8 \%$ and $81.7 \%$, respectively. However, they also showcase through a probing task that BioELMo is a better feature extractor than BioBERT, even though the latter has higher performance when fine tuned on MedNLI. Due to this reason, we take BioELMo as our base architecture and use our enhancements over BioELMo instead of BioBERT.

We also experimented with using fastText and GloVe as our base general embeddings. With addition of Knowledge Graph embeddings and sentiment information, the results showed an absolute improvement from $68.7 \%$ to $73.67 \%$ in case of fastText, and $73.1 \%$ to $74.46 \%$ in case of GloVe. All results are summarized in Table 1.

Training Details: For DistMult, we use word embeddings dimensions to be 100. SGD was used for optimization with an initial learning rate of $10^{-4}$. The batch size was set to 100 . For ESIM, we take the dimension of hidden states of BiLSTMs to be 500. We set the dropout to 0.5 and choose an initial learning rate of $10^{-3}$. We choose a batch size of 32 and run for a maximum of 64 epochs. The training is stopped when the development loss does not decrease after 5 subsequent epochs.

Qualitative Analysis: We explain the efficacy of our model with the help of a few examples. Consider the sentence pair, $p$ : "History of CVA" and $h$ : "patient has history of stroke". In medical terms, 'CVA' means 'Cerebrovascular accident' which is another term for 'stroke'. By Using MetaMap, we are able to find that the preferred term for 'stroke' is 'Cerebrovascular accident' and hence our model classified the sample pair correctly as entailment.

In many cases, our baseline models fail to capture negative sentiment present in the premise or hypothesis. For example, in case of $p$ : Watermelon stomach with gastric varices, without bleed in more than 2 years, $h$ : Patient has no active bleeding, BioELMo predicts this as contradiction, whereas the gold label is entailment. But, by using the negative sentiment, captured by Metamap, for the word 'bleed' in both premise and hypothesis, our model is able predict the label correctly.

On the other hand, even though our model produces promising improvement over state-of-theart performance, there are cases for which our model is not able to classify correctly. For the sen- tence pair $p$ : "She was speaking normally at that time" and $h$ : "The patient has no known normal time where she was speaking normally." contradicting each other, our model predicts this to be entailment. The probable reason could be that, the negative sentiment associated with "speaking normally" is not captured by MetaMap and the noise in MetaMap is percolated further. In another example case, $p$ : "He had no EKG changes and first set of enzymes were negative." and $h$ : "the patient has negative enzymes." our model classifies this pair as entailment while the gold label is neutral. While the premise says that the first set of enzymes was negative, it gives no information about the current state. This leads us to believe that a sense of timeline is extremely important for examples like this which is not already being captured by our model. Taking care of these cases would be our immediate future work.

\section{Conclusion}

In this paper, we showed that knowledge graph embeddings obtained through applying state-ofthe-art model like DistMult from UMLS could be a promising way towards incorporating domain knowledge leading to improved state-of-theart performance for the medical NLI task. We further showed that sentiments of medical concepts can contribute to medical NLI task as well, opening a new direction to be explored further. With the emergence of knowledge graphs in different domains, the proposed approach can be tried out in other domains as well for future exploration.

\section{Acknowledgements}

The authors would like to acknowledge the funding and support from Samsung Research Institute, Delhi (SRID), India.

\section{References}

Lu Shen H Lehman Li-wei Mengling Feng Mohammad Ghassemi Benjamin Moody Peter Szolovits Leo Anthony Celi Alistair EW Johnson, Tom J Pollard and Roger G Mark. 2016. Mimic-iii, a freely accessible critical care database. Scientific data, 3:160035.

A. R. Aronson and F.-M. Lang. 2010. An overview of metamap: Historical perspective and recent advances. J. Amer. Med. Inform. Assoc., 17:229-236.

Xiaodong He Jianfeng Gao Bishan Yang, Wen-tau Yih and Li Deng. 2015. Embedding entities and rela- 
tions for learning and inference in knowledge bases. ICLR.

Olivier Bodenreider. 2004. The unified medical language system (umls): integrating biomedical terminology. Nucleic acids research, 32(suppl_1):D267D270.

Piotr Bojanowski, Edouard Grave, Armand Joulin, and Tomas Mikolov. 2017. Enriching word vectors with subword information. Transactions of the Association for Computational Linguistics, 5:135-146.

Samuel R. Bowman, Gabor Angeli, Christopher Potts, and Christopher D. Manning. 2015. A large annotated corpus for learning natural language inference. In Proceedings of the 2015 Conference on Empirical Methods in Natural Language Processing, pages 632-642, Lisbon, Portugal. Association for Computational Linguistics.

Samuel R Bowman, Raghav Gupta, Jon Gauthier, Christopher D Manning, Abhinav Rastogi, and Christopher Potts. 2016. A fast unified model for parsing and sentence understanding. In 54th Annual Meeting of the Association for Computational Linguistics, ACL 2016. Association for Computational Linguistics (ACL).

Qian Chen, Xiaodan Zhu, Zhen-Hua Ling, Diana Inkpen, and Si Wei. 2018. Neural natural language inference models enhanced with external knowledge. In Proceedings of the 56th Annual Meeting of the Association for Computational Linguistics (Volume 1: Long Papers), pages 2406-2417, Melbourne, Australia. Association for Computational Linguistics.

Qian Chen, Xiaodan Zhu, Zhen-Hua Ling, Si Wei, Hui Jiang, and Diana Inkpen. 2017. Enhanced lstm for natural language inference. In Proceedings of the 55th Annual Meeting of the Association for Computational Linguistics (Volume 1: Long Papers), pages 1657-1668.

Jacob Devlin, Ming-Wei Chang, Kenton Lee, and Kristina Toutanova. 2018. Bert: Pre-training of deep bidirectional transformers for language understanding. In NAACL-HLT.

Qiao Jin, Bhuwan Dhingra, William W. Cohen, and Xinghua Lu. 2019. Probing biomedical embeddings from language models. CoRR, abs/1904.02181.

Rudolf Kadlec, Ondrej Bajgar, and Jan Kleindienst. 2017. Knowledge base completion: Baselines strike back. In Proceedings of the 2nd Workshop on Representation Learning for NLP, pages 69-74, Vancouver, Canada. Association for Computational Linguistics.

Jinhyuk Lee, Wonjin Yoon, Sungdong Kim, Donghyeon Kim, Sunkyu Kim, Chan Ho So, and Jaewoo Kang. 2019. Biobert: a pre-trained biomedical language representation model for biomedical text mining. CoRR, abs/1901.08746.
Mingming Lu, Yu Fang, Fengqi Yan, and Maozhen Li. 2019. Incorporating domain knowledge into natural language inference on clinical texts. IEEE Access.

Tsendsuren Munkhdalai and Hong Yu. 2017. Neural tree indexers for text understanding. In Proceedings of the conference. Association for Computational Linguistics. Meeting, volume 1, page 11. NIH Public Access.

Jeffrey Pennington, Richard Socher, and Christopher Manning. 2014. Glove: Global vectors for word representation. In Proceedings of the 2014 conference on empirical methods in natural language processing (EMNLP), pages 1532-1543.

Matthew Peters, Mark Neumann, Mohit Iyyer, Matt Gardner, Christopher Clark, Kenton Lee, and Luke Zettlemoyer. 2018. Deep contextualized word representations. In Proceedings of the 2018 Conference of the North American Chapter of the Association for Computational Linguistics: Human Language Technologies, Volume 1 (Long Papers), pages 2227-2237.

Alexey Romanov and Chaitanya Shivade. 2018. Lessons from natural language inference in the clinical domain. In Proceedings of the 2018 Conference on Empirical Methods in Natural Language Processing, pages 1586-1596, Brussels, Belgium. Association for Computational Linguistics.

Lei Sha, Baobao Chang, Zhifang Sui, and Sujian Li. 2016. Reading and thinking: Re-read lstm unit for textual entailment recognition. In Proceedings of COLING 2016, the 26th International Conference on Computational Linguistics: Technical Papers, pages 2870-2879.

Yi Tay, Luu Anh Tuan, and Siu Cheung Hui. 2018. Compare, compress and propagate: Enhancing neural architectures with alignment factorization for natural language inference. In Proceedings of EMNLP 2018.

Adina Williams, Nikita Nangia, and Samuel Bowman. 2018. A broad-coverage challenge corpus for sentence understanding through inference. In Proceedings of the 2018 Conference of the North American Chapter of the Association for Computational Linguistics: Human Language Technologies, Volume 1 (Long Papers), pages 1112-1122. 